\title{
小麦/玉米/大豆三熟套作体系中小麦根系分泌特性及氮素吸收研究
}

\author{
雍太文陈小容杨文钰 ${ }^{*}$ 向达兵樊高琼
}

四川农业大学农学院, 四川雅安 625014

\begin{abstract}
摘 要: 为探讨小麦/玉米/大豆多熟套作体系下小麦根系分泌物的分泌特性及其对根系生长环境和植株氮素吸收的 影响, 2006-2008 年连续两个生长季采用田间定位试验, 研究了小麦-大豆、小麦-甘薯、小麦/玉米/大豆和小麦/玉米/ 甘薯 4 种种植模式下小麦根系分泌物的数量与种类、小麦根系生长、土壤水分、土壤氮含量及植株吸氮量的变化特 性。结果表明, 与小麦-大豆和小麦-甘薯两种净作模式及小麦/玉米/甘薯套作模式相比，小麦/玉米/大豆套作降低了开 花期和成熟期小麦生长区的土壤湿度、 $\mathrm{pH}$ 值及 $\mathrm{NO}_{3}{ }^{-}-\mathrm{N}$ 和 $\mathrm{NH}_{4}{ }^{+}-\mathrm{N}$ 的含量, 提高了小麦植株地上部总吸氮量、根系 活力、根干重及土壤总氮含量，并且，开花期小麦根系分泌有机酸总量和可溶性糖含量增加，表现为套作 $>$ 净作，大 豆茬口>甘薯茬口, 边行>中行, 其中以小麦边行处理的分泌量最高。拔节期根系分泌的有机酸, 净作处理以乙酸含量 较高, 占总量的 $47.8 \% \sim 51.6 \%$, 套作处理以柠檬酸含量较高, 占总量的 $31.7 \% \sim 55.1 \%$; 开花期根系分泌的有机酸, 净 作和套作处理均以乙酸含量较高, 占总量的 $33.3 \%$ 78.3\%。小麦/玉米/大豆套作对小麦根系分泌有机酸和可溶性糖有 促进作用, 从而改善了根系生长环境, 提高了小麦对氮素的吸收。
\end{abstract}

关键词: 小麦; 种植模式; 根系分泌物; 氮素吸收; 套作

\section{Root Exudates and Nitrogen Uptake of Wheat in Wheat/Maize/Soybean Relay Cropping System}

\author{
YONG Tai-Wen, CHEN Xiao-Rong, YANG Wen-Yu* , XIANG Da-Bing, and FAN Gao-Qiong \\ College of Agronomy, Sichuan Agricultural University, Ya'an 625014, China
}

\begin{abstract}
Root exudates play a role in adjusting physical and chemical characteristics of soil, improving soil fertility, and facilitating nutrient uptake. Thus, they are used to explaining the mechanism of high nutrient utilization efficiency for plants. In the relay cropping system of wheat/maize/soybean, nitrogen use efficiency appears higher than that in the cropping system of wheat/maize/sweet potato. For explaining the relationship between the high use efficiency of nitrogen and the effects of rhizosphere microenvironment in the wheat/maize/soybean cropping system, the characteristics of wheat root exudates as well as its effects on wheat roots, wheat rhizosphere soil, and nitrogen uptake in wheat were studied in a two-year continuous experiment from 2006 to 2008 with four cropping systems, including two sole cropping systems (wheat-soybean and wheat-sweet potato) and two rely cropping systems (wheat/maize/soybean and wheat/maize/sweet potato). In the wheat/maize/soybean system, the soil moisture, soil $\mathrm{pH}$ and soil quick-acting $\mathrm{N}\left(\mathrm{NO}_{3}{ }^{-}-\mathrm{N}\right.$ and $\left.\mathrm{NH}_{4}{ }^{+}-\mathrm{N}\right)$ content at wheat rhizosphere reduced at both flowering and maturity stages, and the total nitrogen uptake in shoots, root activity, root dry matter weight and soil total nitrogen content of wheat increased. At flowering stage, the total amount of organic acids and the content of soluble sugar of wheat rhizosphere increased in the wheat/maize/soybean system. The total amount of organic acid and the content of soluble sugar of wheat were higher in relay cropping system than in sole cropping system. In the two sole cropping systems, the wheat-soybean mode had higher amount of organic acid and higher content of soluble sugar than wheat-sweet potato. Wheat plants from fringe rows could exude organic acids and higher soluble sugar content than those from central rows, and the amount of root exudates in fringe rows was the highest among all the cropping systems. At jointing stage, the content of acetic acid in the sole cropping systems increased, which accounted for $47.8 \%$ to $51.6 \%$ of the total of organic acid. The content of citric acid in the relay cropping systems increased from $31.7 \%$ to $55.1 \%$ of total organic acid. At flowering stage, the content of acetic acid in sole and relay cropping systems was also promoted from $33.3 \%$ to $78.3 \%$ of the total organic acid. The root exudates were in favor of improving root growth condition, activating soil nutrition, enhancing nitrogen uptake and increasing nitrogen use efficiency in the wheat/maize/soybean relay crop-
\end{abstract}

\footnotetext{
本研究由国家公益性行业(农业)科研专项(nyhyzx07-004-10, 200803028)和国家粮食丰产科技工程专项(2006BAD02A05)资助。

*通讯作者(Corresponding author): 杨文钰, E-mail: wenyu.yang@263.net

第一作者联系方式: E-mail: yongtaiwen@sicau.edu.cn

Received(收稿日期): 2009-08-26; Accepted(接受日期): 2009-12-08.
} 
ping system.

Keywords: Wheat; Cropping system; Root exudates; Nitrogen uptake; Relay cropping

植物根系具有旺盛的代谢活动，并不断向根际 土壤分泌大量的有机物质, 包括黏胶、有机酸、糖、 酚、外酶及多种氨基酸 ${ }^{[1]}$ 。根系分泌物能够调节土 壤微环境, 改善土壤物理性质, 协调肥力因素, 影 响土壤中养分元素的形态及有效性, 刺激土壤中有 益及有害生物的生长繁殖。涂书新和郭志芳 ${ }^{[2]}$ 发现 根系分泌物中的有机酸(主要是草酸)对土壤矿物钾 具有超强的释放作用, 并据此揭示了籽粒苋的高效 富钾机制; 李廷轩等 ${ }^{[3]}$ 和张福锁 ${ }^{[4]}$ 也发现根表钾的 富集与根系分泌物的作用密切相关。根系分泌物与 土壤氮的有效性有关, Rice 和 Pancholy ${ }^{[5]}$ 报道了各种 酚酸化合物能抑制土壤氮的硝化作用; 孙否等 ${ }^{[6]}$ 通 过石英砂培试验发现套作棉花根系分泌物促进了土 壤中磷、钾向有效态转化, 土壤速效氮含量下降, 而 速效磷和速效钾的含量提高, 土壤中酶活性提高, 细菌和真菌数量增多。除大量元素外, 根系分泌物 中有机酸含量的增加还可提高 $\mathrm{Fe} 、 \mathrm{Mn} 、 \mathrm{Cu} 、 \mathrm{Zn}$ 等 微量元素的有效性 ${ }^{[7-8]}$ 。根系分泌物为微生物提供了 碳源, 可提高微生物的繁殖率, 使微生物生长活跃, 尤其是细菌的数量大幅度提高, 利于土壤中氮的转 化 ${ }^{[9]}$ 。此外, 根系分泌的部分糖、有机酸和氨基酸可 直接被植物吸收利用, 从而为植物生命活动提供能 源, 促进植物的生长 ${ }^{[10-11]}$ 。

小麦/玉米/大豆模式是我国西南地区近几年发 展的一种旱地新型高效多熟种植模式 ${ }^{[12]}$, 相对传统 的小麦/玉米/甘薯模式具有明显的增产节肥优势 ${ }^{[13-15]}$ 。 但该模式中作物氮素高效利用特性与套作系统中根 际微生态效应的关系尚不清楚, 尤其是多熟套作对 作物根系分泌物的影响鲜见报道。本文以该模式中 的小麦为研究对象, 以小麦/玉米/甘薯模式为对照, 通过连续两年度的定位试验, 分析了在不同种植模 式中小麦微区轮作条件下的根系分泌物、小麦根系 生长、土壤氮含量、土壤环境及植株吸氮量的变化 特征, 并探讨了根系分泌物与小麦/玉米/大豆套作 体系下小麦氮素吸收特性的关系。

\section{1 材料与方法}

\section{1 作物品种及田间设计}

供试小麦、玉米、大豆、甘薯品种依次为川农 18 (四川农业大学农学院)、川单 418 (四川农业大学 玉米所)、贡选 1 号(自贡市农业科学研究所)、川薯
164 (四川省农业科学院作物研究所)。试验于 2006 年 11 月至 2008 年 5 月在四川农业大学教学农场进 行, 单因素(种植模式)随机区组设计, 设两个净作处 理, 即小麦-大豆(A1)和小麦-甘薯(A2)连作模式, 及 两个套作处理, 即小麦/玉米/大豆(A3)和小麦/玉米/ 甘薯(A4)套作模式, 每种模式均 3 次重复, 小区面积 $42 \mathrm{~m}^{2}$ 。套作小区套种 3 带, 带宽 $2 \mathrm{~m}$, 其中小麦(或 大豆、甘薯)和玉米幅宽各 $1 \mathrm{~m}$, 每幅小麦播种 5 行, 幅内行距 $0.2 \mathrm{~m}$, 穴距 $0.1 \mathrm{~m}$, 每穴定苗 4 株。每公顷 小麦施肥量为纯氮 $84 \mathrm{~kg} 、 \mathrm{P}_{2} \mathrm{O}_{5} 67.5 \mathrm{~kg} 、 \mathrm{~K}_{2} \mathrm{O} 90 \mathrm{~kg}$; 净作小区种植密度和施肥水平与套作小区相同。

两年度播种时间相同，均为小麦 11 月 4 日、玉 米 3 月 20 日、大豆或甘薯 5 月 22 日。2007-2008 年度小麦换茬轮作, 即套作时小麦种在玉米茬口上, 净作时小麦种在大豆或甘薯茬口上。

\section{2 取样方法}

于小麦拔节期、开花期和成熟期, 采用传统挖 掘法取植株、根系与土壤样品, 套作处理分边 2 行 和中 3 行分别取样, 净作处理取中间 3 行。每小区 随机取长势一致样段 $(0.2 \mathrm{~m}$ 宽)两段, 每段取连续 2 穴, 套作边行取 32 株, 中行与净作取 48 株。取样土 方规格, 边行为 $10 \mathrm{~cm} \times 20 \mathrm{~cm} \times 40 \mathrm{~cm}$, 净作和中行 为 $30 \mathrm{~cm} \times 20 \mathrm{~cm} \times 40 \mathrm{~cm}$ 。各种作物均在籽粒完全 成熟时进行收获。

\section{3 样品分析方法}

1.3.1 土壤样品 从挖出土方中抖落与根系松散 结合的土体土作为待测土, 将两个样段的土样混匀 按 4 分法取样, 过 $0.45 \mathrm{~mm}$ 钢篮除去杂物, 取 $10 \mathrm{~g}$ 土保存在 $4^{\circ} \mathrm{C}$ 冰箱内, 用于土壤 $\mathrm{NH}_{4}{ }^{+}-\mathrm{N} 、 \mathrm{NO}_{3}{ }^{-}-\mathrm{N}$ 含 量的测定, 剩下的自然风干, 用于土壤湿度、 $\mathrm{pH}$ 值 和总氮含量的测定。

采用烘干法 ${ }^{[16]}$ 测定土壤湿度; 用 PHS-29A 型酸 度计测定土壤 $\mathrm{pH}$ 值 ${ }^{[16]}$, 水土比为 2.5: 1.0 ; 采用凯 氏定氮法 ${ }^{[16]}$ 测定土壤总氮含量。称取 $5.00 \mathrm{~g}$ 鲜土置 振荡瓶中, 加 $1 \mathrm{~mol} \mathrm{~L}^{-1} \mathrm{KCl}$ 溶液 $50 \mathrm{~mL}$, 于恒温气 浴摇床 $120 \mathrm{r} \mathrm{min}$ 下振荡 $60 \mathrm{~min}$ 后, 取下过滤液, 保存在塑料瓶中, 置 $4^{\circ} \mathrm{C}$ 冰箱冷藏, 1 周内用 INTEGRAL Futura 连续流动分析仪(ALLIANCE 公司, 法 国)测定土壤 $\mathrm{NH}_{4}{ }^{+}-\mathrm{N}$ 和 $\mathrm{NO}_{3}{ }^{-}-\mathrm{N}$ 含量。

1.3.2 根系分泌物参照李廷轩等 ${ }^{[3]}$ 、胡学玉 等 ${ }^{[17]}$ 对根系分泌物的测定方法, 在 2007-2008 年度, 
从挖取土方中选取根系完好的植株 6 株, 先用自来水 冲洗根部 5 10 次, 然后用蒸馏水清洗 3 5 次, 再用 重蒸水清洗 2 3 次。用滤纸吸干根表面水分后, 将 根系置于装有 $300 \mathrm{~mL}$ 重蒸水的塑料烧杯中, 用海绵 固定植株, 并培养于人工气候箱中 $\left(\right.$ 温度 $25^{\circ} \mathrm{C}$, 相对 湿度为 $70 \%$, 光照强度为 $600 \mu \mathrm{mol} \mathrm{m} \mathrm{m}^{-2} \mathrm{~s}^{-1}$ )。 $6 \mathrm{~h}$ 后 收集溶液，并咜于棕色瓶中，保存在 $4^{\circ} \mathrm{C}$ 冰箱内。将 收集到的溶液先过两层滤纸, 再过 $0.45 \mu \mathrm{m}$ 滤膜, 除 去碎屑杂物, 以旋转蒸发血 $40^{\circ} \mathrm{C}$ 减压浓缩, 定容至 $10 \mathrm{~mL}$ ，䛎于 $-20^{\circ} \mathrm{C}$ 。

采用 1100 Serzes 型高效液相色谱仪(Agilent 公 司, 美国)测定根系分泌物中有机酸的种类及含量, 紫外检测波长为 $214 \mathrm{~nm}$, 检测条件为柱温 $20^{\circ} \mathrm{C}$, 流 速 $1.0 \mathrm{~mL} \mathrm{~min}{ }^{-1}$, 进样量 $50 \mu \mathrm{L}$, 流动相为超纯水: 乙腈 $(V / V)=96.5: 3.5$, 等度分离。有机酸标准样品 为甲酸、乳酸、乙酸、柠檬酸、琥珀酸和延胡索酸 等标准品的混合液, 浓度分别为 $2.8607 、 2.5606$ 、 $5.3204 、 5.4402 、 5.5709$ 和 $0.0731 \mathrm{~g} \mathrm{~L}^{-1}$ 。用葸酮比 色法测定分泌物中可溶性糖 ${ }^{[18]}$ 。

1.3.3 植株样品 将土方装入尼龙网袋, 水浸泡, 快速冲洗干净根系, 并将泥水过 $0.45 \mathrm{~mm}$ 钢篮, 收 集断根, 去掉枯死的叶片, 用吸水纸吸干植株水滴, 分离出根部和植株地上部。地上部分秸秆和籽粒于 $105^{\circ} \mathrm{C}$ 下杀青 $30 \mathrm{~min}$ 后 $70^{\circ} \mathrm{C}$ 烘干, 用百分之一的 电子天平称重后粉碎, 用凯式定氮法测定全氮含 量 ${ }^{[16]}$ 。2007-2008 年度取样后, 称取所有样段全部 植株的根的鲜重, 混匀后一部分根用于 TTC 法测定 根系活力 ${ }^{[18]}$, 另一部分用于测定水分含量, 计算出 根系干重。

\section{4 统计分析}

使用 Microsoft Excel 2003 软件处理试验中的图 表, SPSS16.0 软件进行方差分析、LSD 多重比较及 相关分析。

\section{2 结果与分析}

\section{1 种植模式对根系分泌物的影响}

2.1.1 有机酸 种植模式对小麦根系分泌物中各 类有机酸含量及总量均有显著影响(表 1)。在拔节期 与开花期, 有机酸分泌总量表现为套作 > 净作, 大豆 茬口 $>$ 甘薯茬口, 边行 $>$ 中行, 以 A3 处理边行最高, 显著高于 $\mathrm{A} 3$ 处理中行及净作和 $\mathrm{A} 4$ 处理, 尤其是开 花期, 仅 $\mathrm{A} 3$ 处理边行的有机酸分泌量比拔节期高 $15.1 \%$, 其他各处理的有机酸总量均出现降低趋势;
A3 边行比净作的 A1 和 A2 处理分别高 2.9 倍和 4.9 倍，比套作的 A3 中行、A4 边行和 A4 中行分别高 1.7、0.8 和 2.3 倍。

从有机酸种类看, 以乙酸、柠檬酸和琥珀酸为 主，在种植方式间及生育期间差异明显(表 1)。在拔 节期, 净作处理以乙酸分泌量较大, 占有机酸总量 的 47.8\% 51.6\%, 表现为 $\mathrm{A} 1>\mathrm{A} 2>\mathrm{A} 3$ 边行 $>\mathrm{A} 4$ 边行 $>\mathrm{A} 3$ 中行 $>\mathrm{A} 4$ 中行; 套作处理以柠檬酸含量较高, 占有机酸总量的 $31.7 \% \sim 55.1 \%$, 表现为 $\mathrm{A} 3$ 边行 $>\mathrm{A} 3$ 中行 $>A 4$ 边行 $>A 4$ 中行 $>A 1>A 2$ 。在开花期, 无论净 作还是套作均以乙酸含量较高, 占有机酸总量的 $33.3 \% \sim 78.3 \%$, 以 A3 边行最高; 表现为套作 $>$ 净作, 大豆茬口 $>$ 甘薯茬口，边行 $>$ 中行。与拔节期相比，开 花期除套作处理分泌的乙酸含量较大幅度增加外, 其他处理的乙酸含量及所有处理的柠檬酸和琥珀酸 含量均有不同程度的降低。甲酸在小麦根系分泌有 机酸中所占比重较小，且变化无规律。拔节期仅在 套作中检测到其含量，以甘薯茬口的较高; 开花期 则仅在净作中检测到其含量, 以大豆茬口的较高。 延胡索酸的含量较低, 各处理间和生育期间的差异 不显著。

2.1.2 可溶性糖 种植模式对小麦根系分泌物中 可溶性糖含量有显著影响(表 1), 且对小麦与玉米共 生前后的影响规律不一致。共生前的拔节期，根系 分泌可溶性糖含量表现为套作 $>$ 净作, 甘薯茬口 $>$ 大 豆茬口, 中行>边行, 以 $\mathrm{A} 4$ 中行最高, 比净作 A1 和 $\mathrm{A} 2$ 处理分别高 1.1 倍和 1.1 倍, 比套作处理 A3 边行、 A3 中行和 A4 边行分别高 $96.6 \%$ 、48.1\%和 $8.5 \%$ 。 随生育进程的推进，除 A2 处理外，其他处理的小麦 根系分泌物中的可溶性糖含量均呈增加趋势, 开花 期呈现与有机酸含量一致的趋势，即套作 $>$ 净作，大 豆茬口 $>$ 甘薯茬口, 边行 $>$ 中行, 以 $\mathrm{A} 3$ 边行最高, 分 别比 A1、A2、A3 中行、A4 边行和 A4 中行高 $86.5 \%$ 、 $294.7 \% 、 81.5 \%$ 、30.1\%和 $95.6 \%$ 。

\section{2 种植模式对小麦根系生长的影响}

种植模式对小麦根系干重有显著影响，拔节期 与开花期均为大豆茬口的单株根系干重高于甘薯茬 口，相同茬口下套作处理高于净作; 从边、中行效应 来看, 拔节期为中行的单株根系干重高于边行, 但 随生育进程的推进，小麦对玉米生长区养分的吸收 能力增强, 边行根系的生长速度逐渐加快, 开花期 边行的单株根系干重已显著高于中行, 以 A3 处理 边行最高, 分别比 A3 处理中行、 $\mathrm{A} 4$ 处理边行和中 
表 $1 \quad 2007-2008$ 年度不同种植模式小麦根系分泌物中的有机酸和可溶性糖含量

Table 1 Contents of organic acids and soluble sugar in root exudates of wheat under various cropping systems (2007-2008)

\begin{tabular}{|c|c|c|c|c|c|c|c|}
\hline \multirow{2}{*}{$\begin{array}{c}\text { 处理 } \\
\text { Treatment }\end{array}$} & \multicolumn{6}{|c|}{ 有机酸含量 Contents of organic acids $\left(\mathrm{mg}\right.$ plant $\left.^{-1} \mathrm{~h}^{-1}\right)$} & \multirow{2}{*}{$\begin{array}{c}\text { 可溶性糖含量 } \\
\text { Content of soluble sugar } \\
\left(\mathrm{mg} \text { plant }{ }^{-1} \mathrm{~h}^{-1}\right)\end{array}$} \\
\hline & $\begin{array}{c}\text { 甲酸 } \\
\text { Formic }\end{array}$ & $\begin{array}{c}\text { 乙酸 } \\
\text { Acetic }\end{array}$ & $\begin{array}{c}\text { 柠檬酸 } \\
\text { Citric }\end{array}$ & $\begin{array}{c}\text { 琥珀酸 } \\
\text { Succinic }\end{array}$ & $\begin{array}{c}\text { 延胡索酸 } \\
\text { Fumaric }\end{array}$ & $\begin{array}{l}\text { 总量 } \\
\text { Total }\end{array}$ & \\
\hline \multicolumn{8}{|c|}{ 拔节期 Jointing stage } \\
\hline A1 & - & $0.863 \mathrm{a}$ & $0.564 \mathrm{~b}$ & $0.375 \mathrm{~b}$ & $0.001 \mathrm{a}$ & $1.803 \mathrm{c}$ & $0.660 \mathrm{~b}$ \\
\hline A2 & - & $0.785 \mathrm{ab}$ & $0.347 \mathrm{c}$ & $0.387 \mathrm{~b}$ & $0.001 \mathrm{a}$ & $1.520 \mathrm{~d}$ & $0.677 \mathrm{~b}$ \\
\hline A3-1 & $0.321 \mathrm{a}$ & $0.687 \mathrm{bc}$ & $1.766 \mathrm{a}$ & $0.429 \mathrm{ab}$ & $0.001 \mathrm{a}$ & $3.205 \mathrm{a}$ & $0.705 \mathrm{~b}$ \\
\hline A3-2 & $0.218 \mathrm{~b}$ & $0.596 \mathrm{~cd}$ & $0.745 \mathrm{~b}$ & $0.209 \mathrm{c}$ & $0.001 \mathrm{a}$ & $1.770 \mathrm{~cd}$ & $0.937 \mathrm{ab}$ \\
\hline A4-1 & $0.422 \mathrm{a}$ & $0.628 \mathrm{c}$ & $0.738 \mathrm{~b}$ & $0.541 \mathrm{a}$ & $0.001 \mathrm{a}$ & $2.330 \mathrm{~b}$ & $1.278 \mathrm{a}$ \\
\hline A4-2 & $0.355 \mathrm{a}$ & $0.517 \mathrm{~d}$ & $0.654 \mathrm{~b}$ & $0.342 \mathrm{~b}$ & $0.001 \mathrm{a}$ & $1.868 \mathrm{c}$ & $1.387 \mathrm{a}$ \\
\hline \multicolumn{8}{|c|}{ 开花期 Flowering stage } \\
\hline A1 & $0.201 \mathrm{a}$ & $0.317 \mathrm{~d}$ & $0.259 \mathrm{bc}$ & $0.172 \mathrm{c}$ & $0.001 \mathrm{bc}$ & $0.952 \mathrm{de}$ & $1.338 \mathrm{c}$ \\
\hline A2 & $0.181 \mathrm{~b}$ & $0.273 \mathrm{~d}$ & $0.056 \mathrm{~d}$ & $0.116 \mathrm{c}$ & $0.001 \mathrm{bc}$ & $0.626 \mathrm{e}$ & $0.632 \mathrm{~d}$ \\
\hline A3-1 & - & $2.546 \mathrm{a}$ & $0.441 \mathrm{a}$ & $0.699 \mathrm{a}$ & $0.003 \mathrm{a}$ & $3.688 \mathrm{a}$ & $2.496 \mathrm{a}$ \\
\hline A3-2 & - & $1.082 \mathrm{bc}$ & $0.167 \mathrm{c}$ & $0.133 \mathrm{c}$ & $0.001 \mathrm{bc}$ & $1.383 \mathrm{c}$ & $1.375 \mathrm{bc}$ \\
\hline A4-1 & - & $1.116 \mathrm{~b}$ & $0.365 \mathrm{ab}$ & $0.592 \mathrm{~b}$ & $0.002 \mathrm{~b}$ & $2.074 \mathrm{~b}$ & $1.918 \mathrm{~b}$ \\
\hline A4-2 & - & $0.843 \mathrm{c}$ & $0.152 \mathrm{~cd}$ & $0.114 \mathrm{c}$ & $0.001 \mathrm{bc}$ & $1.110 \mathrm{~cd}$ & $1.276 \mathrm{c}$ \\
\hline
\end{tabular}

$\mathrm{A} 1$ : 小麦-大豆连作; $\mathrm{A} 2$ : 小麦-甘薯连作; A3-1：小麦/玉米/大豆套作边行; A3-2: 小麦/玉米/大豆套作中行; A4-1: 小麦/玉米/甘 薯套作边行; A4-2: 小麦/玉米/甘薯套作中行。表中数据为 3 次重复的平均值。同一列中标以不同字母的值差异达 0.05 显著水平。“一” 表示含量未检出。

A1: wheat-soybean cropping system; A2: wheat-sweet potato cropping system; A3-1: fringe rows of wheat/maize/soybean cropping system; A3-2: central rows of wheat/maize/soybean cropping system; A4-1: fringe rows of wheat/maize/sweet potato cropping system; A4-2: central rows of wheat/maize/sweet potato cropping system. Data are the averages of three replicates. Values followed by a different letter within each column are significantly different at the 0.05 probability level. "-” denotes content undetectable.

表 $22007-2008$ 年度不同种植模式的小麦根系干物重和根系活力

Table 2 Root dry matter weight and root activity of wheat under various cropping systems (2007-2008)

\begin{tabular}{cccccc}
\hline \multirow{2}{*}{$\begin{array}{c}\text { 处理 } \\
\text { Treatment }\end{array}$} & \multicolumn{2}{c}{ 根系干物重 Root dry matter weight $\left(\mathrm{g} \mathrm{plant}{ }^{-1}\right)$} & & \multicolumn{2}{c}{ 根系活力 Root activity $\left(\mu \mathrm{g} \mathrm{g} \mathrm{g}^{-1}\right)$} \\
\cline { 2 - 3 } \cline { 5 - 6 } A1 & 拔节期 Jointing stage & 开花期 Flowering stage & & 拔节期 Jointing stage & 开花期 Flowering stage \\
\hline A2 & $0.237 \mathrm{a}$ & $0.331 \mathrm{ab}$ & & $351.326 \mathrm{a}$ & $67.269 \mathrm{ab}$ \\
A3-1 & $0.217 \mathrm{a}$ & $0.252 \mathrm{c}$ & & $174.182 \mathrm{~b}$ & $57.764 \mathrm{~b}$ \\
A3-2 & $0.271 \mathrm{a}$ & $0.352 \mathrm{a}$ & & $267.811 \mathrm{ab}$ & $80.027 \mathrm{a}$ \\
A4-1 & $0.284 \mathrm{a}$ & $0.320 \mathrm{ab}$ & & $172.156 \mathrm{~b}$ & $69.863 \mathrm{ab}$ \\
A4-2 & $0.241 \mathrm{a}$ & $0.312 \mathrm{ab}$ & & $193.953 \mathrm{~b}$ & $65.725 \mathrm{~b}$ \\
\hline
\end{tabular}

A1: 小麦-大豆连作; A2: 小麦-甘薯连作; A3-1: 小麦/玉米/大豆套作边行; A3-2: 小麦/玉米/大豆套作中行; A4-1: 小麦/玉米/甘 薯套作边行; A4-2: 小麦/玉米/甘薯套作中行。表中数据为 3 次重复的平均值。同一列中标以不同字母的值差异达 0.05 显著水平。

A1: wheat-soybean cropping system; A2: wheat-sweet potato cropping system; A3-1: fringe rows of wheat/maize/soybean cropping system; A3-2: central rows of wheat/maize/soybean cropping system; A4-1: fringe rows of wheat/maize/sweet potato cropping system; A4-2: central rows of wheat/maize/sweet potato cropping system. Data are the averages of three replicates. Values followed by a different letter within each column are significantly different at the 0.05 probability level.

行、A1 和 A2 处理高 $10.0 \% 、 12.8 \% 、 22.6 \% 、 6.3 \%$ 和 $39.7 \%$ (表 2)。

不同茬口比，拔节期与开花期均为大豆茬口的 单株根系活力显著高于甘薯茬口，尤其是小麦拔节 期(此时玉米尚未播种), 二者相差 2.0 倍; 净、套作 模式比, 小麦拔节期呈现相同茬口下净作的根系活 力高于套作, 而开花期(小麦与玉米共生), 表现为相
同茬口下套作的根系活力高于净作, 且边行优于中 行, 以 $\mathrm{A} 3$ 处理边行为最高, 分别比 $\mathrm{A} 3$ 处理中行、 A4 处理边行和中行、A1 和 A2 处理高 $14.5 \%$ 、21.8\%、 $124.3 \%$ 、18.9\%和 $38.5 \%$ (表 2 )。

\section{3 种植模式对土壤湿度及 $\mathbf{p H}$ 的影响}

2.3.1 土壤湿度 2006-2007 年度, 开花期与成 熟期均表现为净作土壤湿度显著高于套作, 成熟期 
净作的土壤湿度比套作高 $12.2 \%$ 21.5\%。20072008 年度, 各生育期均表现为净作的土壤湿度高于 套作, 但不同茬口的变化规律不一致, 净作中大豆 茬口的土壤湿度略低于甘薯茬口, 差异不显著; 套 作体系则表现为 $\mathrm{A} 3$ 处理的土壤湿度高于 $\mathrm{A} 4$ 处理, 且边行的土壤湿度均低于相应处理的中行以及两个 净作处理, 尤其是在开花期, 此时 A3 和 $\mathrm{A} 4$ 处理的 边行分别比中行低 $23.8 \%$ 和 $17.4 \%$ (表 3)。

\subsection{2 土壤 $\mathrm{pH}$ 各种植模式下小麦根际土壤 $\mathrm{pH}$} 变化不大, 介于 6.2 6.8 之间; 随生育进程的推进, $\mathrm{pH}$ 呈降低趋势(图 1)。净作土壤 $\mathrm{pH}$ 值略高于套作, 大豆茬口低于甘薯茬口, 边行低于中行和净作, 各 处理中以 $\mathrm{A} 3$ 边行土壤 $\mathrm{pH}$ 值最低。这可能与套作模 式下根系分泌有机酸总量增多有关。

\section{4 种植模式对植株吸氮量的影响}

2006-2007 年度, A3 和 A4 处理的茎叶吸氮量 显著高于 $\mathrm{A} 1$ 和 $\mathrm{A} 2$ 处理, 开花期平均高 $33.9 \%$, 成 熟期平均高 $14.4 \%$; 成熟期籽粒吸氮量和地上部总 吸氮量套作比净作分别高 $41.3 \%$ 60.5\%和 $24.2 \%$ $34.2 \%$ 。2007-2008 年度, 由于受轮作与套作效应的 影响, 小麦植株氮素吸收特性发生了变化, 各生育 期小麦吸氮量表现为大豆茬口高于甘薯茬口, 相同 茬口下套作处理高于净作处理。从套作处理的边、 中行效应来看, 各生育期 A3 和 A4 处理边行的茎叶 和籽粒吸氮量均高于相应处理的中行及净作处理, 以 $\mathrm{A} 3$ 处理的边行最高, 其中籽粒吸氮量 $\mathrm{A} 3$ 处理边 行比 A1 和 A2 处理分别高 35.8\%和 61.9\%, 其地上部
总吸氮量也比 A1 和 A2 处理高 18.0\%和 31.0\% (表 4)。 2.5 种植模式对土壤氮含量的影响

2.5.1 土壤总氮含量 小麦土壤总氮含量随生育 期呈降低趋势, 在小麦与玉米共生前表现为净作高 于套作, 小麦与玉米共生后套作高于净作, 尤其是 $2007-2008$ 年度换茬轮作后, 这种变化尤为明显(表 5)。在种植玉米前的拔节期, 土壤总氮含量为净作高 于套作, 大豆茬口略低于甘薯茬口, 其原因可能是 该时期土壤中氮素矿化与硝化速度较慢, 而大豆茬 口在播种小麦前土壤总氮含量较高, 有利于植株吸 收, 致使土壤总氮相对降低; 随着小麦与玉米的套 种共生, 小麦开始竞争吸收玉米土壤中的氮, 使得 套作小麦土壤中氮浓度降低速度减慢, 最终表现出 套作小麦的土壤总氮含量高于净作, 边行高于中行, 大豆茬口高于甘薯茬口。成熟期小麦土壤总氮含量, $\mathrm{A} 3$ 处理边行比 A1、A2 和 A4 处理边行及中行分别 高 $4.7 \% 、 7.3 \% 、 2.5 \%$ 和 $6.9 \%$ (表 5 )。

2.5.2 土壤 $\mathrm{NO}_{3}{ }^{-}-\mathrm{N}$ 和 $\mathrm{NH}_{4}{ }^{+}-\mathrm{N}$ 含量 随生育进程 的推进, 硝化作用增强, 土壤中 $\mathrm{NO}_{3}{ }^{-}-\mathrm{N}$ 含量逐渐增 高, 当开花期植株吸氮量达到高峰时, 土壤中的 $\mathrm{NO}_{3}{ }^{-}-\mathrm{N}$ 含量也随之达到高峰, 之后开始降低。两年 度试验均表现为净作土壤 $\mathrm{NO}_{3}^{-}-\mathrm{N}$ 含量显著高于套 作处理(表 6), 说明净作小麦从土壤中吸收 $\mathrm{NO}_{3}{ }^{-}-\mathrm{N}$ 量较低, 使植株吸氮量低于套作; 大豆茬口的 $\mathrm{NO}_{3}{ }^{-}-\mathrm{N}$ 含量低于甘薯茬口, 边行 $\mathrm{NO}_{3}{ }^{-}-\mathrm{N}$ 含量低于 中行(表 6), 说明 A3 套作体系通过根系分泌物对小 麦土壤环境的改善, 加强了对无机氮的吸收能力,

表 3 不同种植模式的小麦土壤湿度

Table 3 Soil moisture of wheat under various cropping systems (\%)

\begin{tabular}{|c|c|c|c|c|c|}
\hline \multirow{2}{*}{$\begin{array}{c}\text { 处理 } \\
\text { Treatment }\end{array}$} & \multicolumn{2}{|c|}{ 2006-2007 } & \multicolumn{3}{|c|}{$2007-2008$} \\
\hline & 开花期 Flowering & 成熟期 Maturity & 拔节期 Jointing & 开花期 Flowering & 成熟期 Maturity \\
\hline A1 & $21.5 \mathrm{ab}$ & $26.2 \mathrm{a}$ & $26.5 \mathrm{a}$ & $17.6 \mathrm{a}$ & $23.7 \mathrm{a}$ \\
\hline A2 & $23.2 \mathrm{a}$ & $27.2 \mathrm{a}$ & $26.9 \mathrm{~b}$ & $17.9 \mathrm{a}$ & $23.4 \mathrm{a}$ \\
\hline A3-1 & - & - & $25.5 \mathrm{c}$ & $13.4 \mathrm{~b}$ & $21.4 \mathrm{ab}$ \\
\hline A3-2 & $20.4 \mathrm{bc}$ & $23.4 \mathrm{~b}$ & $26.1 \mathrm{bc}$ & $17.6 \mathrm{a}$ & $23.4 \mathrm{a}$ \\
\hline A4-1 & - & - & $23.9 \mathrm{~d}$ & $13.3 \mathrm{~b}$ & $17.2 \mathrm{~b}$ \\
\hline A4-2 & $18.5 \mathrm{c}$ & $22.4 \mathrm{~b}$ & $22.5 \mathrm{e}$ & $16.1 \mathrm{a}$ & $19.8 \mathrm{ab}$ \\
\hline
\end{tabular}

A1：小麦-大豆连作; A2：小麦-甘薯连作; A3-1：小麦/玉米/大豆套作边行; A3-2：小麦/玉米/大豆套作中行; A4-1：小麦/玉米/甘 薯套作边行; A4-2: 小麦/玉米/甘薯套作中行。表中数据为 3 次重复的平均值。同一列中标以不同字母的值差异达 0.05 显著水平。 2006-2007 年度, 套作处理未按边行和中行分别取样, 因此只有一组数据。

A1: wheat-soybean cropping system; A2: wheat-sweet potato cropping system; A3-1: fringe rows of wheat/maize/soybean cropping system; A3-2: central rows of wheat/maize/soybean cropping system; A4-1: fringe rows of wheat $/ \mathrm{maize} / \mathrm{sweet}$ potato cropping system; A4-2: central rows of wheat/maize/sweet potato cropping system. Data are the averages of three replicates. Values followed by a different letter within each column are significantly different at the 0.05 probability level. In 2006-2007, wheat plants were sampled only from central rows of treatments $\mathrm{A} 3$ and $\mathrm{A} 4$. 

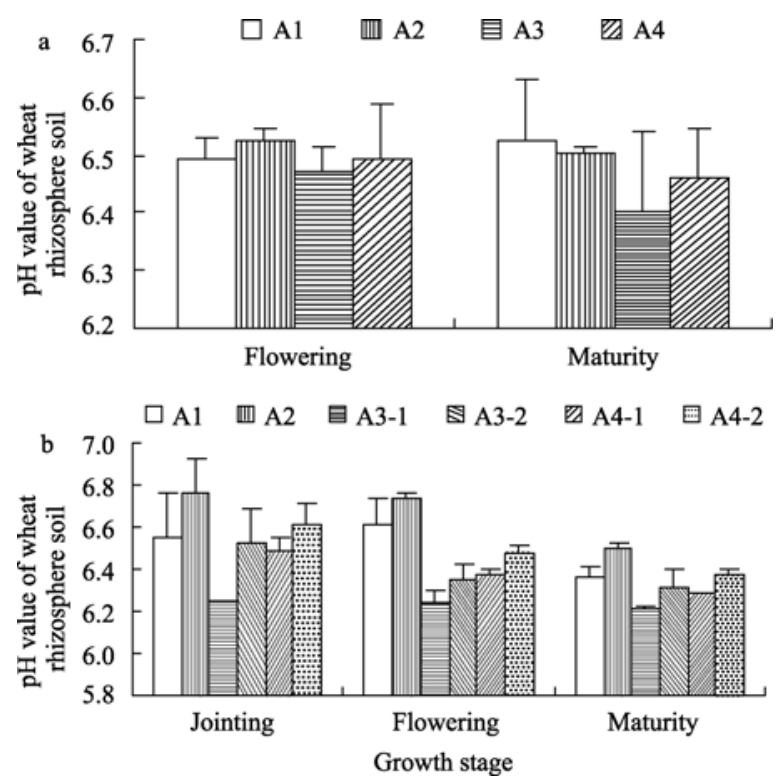

图 1 不同种植模式的小麦根际土壤 $\mathrm{pH}$ 值

Fig. 1 pH value of wheat rhizosphere soil under various cropping systems

$\mathrm{A} 1$ : 小麦-大豆连作; $\mathrm{A} 2$ : 小麦-甘薯连作; $\mathrm{A} 3$ : 小麦/玉米/大豆套 作, 其中 A3-1 和 A3-2 分别为该处理的边行和中行; A4: 小麦/ 玉米/甘薯套作, 其中 A4-1 和 A4-2 分别为该处理的边行和中行。 数据为 3 次重复的平均值 \pm 标准差。 $\mathrm{a}: 2006-2007$ 年度; $\mathrm{b}$ : 2007-2008 年度。

A1: wheat-soybean cropping system; A2: wheat-sweet potato cropping system; A3: wheat/maize/soybean cropping system, of which A3-1 and A3-2 represent fringe and central rows, respectively; A4: wheat/maize/sweet potato cropping system, of which A4-1 and A4-2 represent fringe and central rows, respectively. Columns are shown in mean $\pm S D$. a: 2006-2007; b: 2007-2008.
降低了土壤中 $\mathrm{NO}_{3}{ }^{-}-\mathrm{N}$ 的富积, 不仅有利提高作物的 产量, 也利于提高氮肥利用效率, 减少对环境的污 染。土壤 $\mathrm{NH}_{4}{ }^{+}-\mathrm{N}$ 含量与 $\mathrm{NO}_{3}{ }^{-}-\mathrm{N}$ 含量呈现一致规律, 即净作略高于套作，大豆茬口显著低于甘薯茬口， 边行显著低于相应处理的中行及净作处理。但 $\mathrm{NH}_{4}{ }^{+}-\mathrm{N}$ 含量在各生育期表现不一致，在吸肥高峰的 开花期含量最高, 且差异最明显, 其他生育期差异 不显著(表 6), 这可能与该时期矿化作用最强和蛋白 酶活性最高有关。因此, A3 套作模式不仅有效提高 了土壤总氮含量, 还通过降低土壤中 $\mathrm{NO}_{3}{ }^{-}-\mathrm{N} 、 \mathrm{NH}_{4}{ }^{+}-$ $\mathrm{N}$ 等无机氮含量, 减轻氮流失, 提高氮的有效性。

\section{3 讨论}

\section{1 茬口与套作效应对小麦根系分泌物的影响}

营养胁迫条件下，植物可以通过调节其自身的 生命活动过程来适应环境 ${ }^{[19-20]}$ 。作物种类、基因型 以及营养状况等都可影响根系分泌物的数量和种

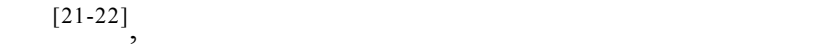
量也存在差异 ${ }^{[23]}$ 。在间套作体系中，小麦与玉米间 作, 根系分泌物中酸的种类明显增加, 而植株体内 和根系中的有机酸种类和数量却降低; 其中, 单作 小麦主要分泌苹果酸和柠檬酸, 间作小麦以分泌草 酸和柠檬酸为主 ${ }^{[24]}$ 。在麦棉套作系统中, 棉苗根系 分泌物对土壤速效养分含量及土壤微生物有明显的 促进作用 ${ }^{[6]}$ 。利用 ${ }^{14} \mathrm{C}$ 同位素示踪技术, 发现在玉米

表 4 不同种植模式的小麦植株吸氮量

Table 4 Nitrogen uptake of wheat under various cropping systems $\left(\mathrm{kg} \mathrm{hm}^{-2}\right)$

\begin{tabular}{|c|c|c|c|c|c|c|c|c|c|}
\hline \multirow{3}{*}{$\begin{array}{c}\text { 处理 } \\
\text { Treatment }\end{array}$} & \multicolumn{4}{|c|}{$2006-2007$} & \multicolumn{5}{|c|}{$2007-2008$} \\
\hline & \multirow{2}{*}{$\begin{array}{c}\text { 开花期茎叶吸氮 } \\
\mathrm{N} \text { uptake in shoot } \\
\text { at flowering }\end{array}$} & \multicolumn{3}{|c|}{ 成熟期吸氮 $N$ uptake at maturity } & \multirow{2}{*}{$\begin{array}{c}\text { 拔节期茎叶吸氮 } \\
\mathrm{N} \text { uptake in shoot } \\
\text { at jointing }\end{array}$} & \multirow{2}{*}{$\begin{array}{c}\text { 开花期茎叶吸氮 } \\
\mathrm{N} \text { uptake in shoot } \\
\text { at flowering }\end{array}$} & \multicolumn{3}{|c|}{ 成熟期吸氮 $N$ uptake at maturity } \\
\hline & & $\begin{array}{l}\text { 茎叶 } \\
\text { Shoot }\end{array}$ & $\begin{array}{l}\text { 籽粒 } \\
\text { Grain }\end{array}$ & $\begin{array}{l}\text { 总量 } \\
\text { Total }\end{array}$ & & & $\begin{array}{l}\text { 茎叶 } \\
\text { Shoot }\end{array}$ & $\begin{array}{l}\text { 籽粒 } \\
\text { Grain }\end{array}$ & $\begin{array}{l}\text { 总量 } \\
\text { Total }\end{array}$ \\
\hline $\mathrm{A} 1$ & $172.2 \mathrm{~b}$ & $157.9 \mathrm{~b}$ & $88.8 \mathrm{~b}$ & $246.7 \mathrm{bc}$ & $199.5 \mathrm{ab}$ & $262.9 \mathrm{ab}$ & $187.8 \mathrm{ab}$ & $85.5 \mathrm{c}$ & $273.3 \mathrm{bc}$ \\
\hline $\mathrm{A} 2$ & $177.9 \mathrm{~b}$ & $152.2 \mathrm{~b}$ & $76.1 \mathrm{~b}$ & $228.3 \mathrm{c}$ & $176.0 \mathrm{~b}$ & $228.3 \mathrm{~b}$ & $174.5 \mathrm{~b}$ & $71.7 \mathrm{~d}$ & $246.2 \mathrm{c}$ \\
\hline A3-1 & - & - & - & - & $219.8 \mathrm{a}$ & $300.8 \mathrm{a}$ & $206.4 \mathrm{a}$ & $116.1 \mathrm{a}$ & $322.5 \mathrm{a}$ \\
\hline A3-2 & $225.4 \mathrm{a}$ & $180.9 \mathrm{a}$ & $125.5 \mathrm{a}$ & $306.3 \mathrm{a}$ & $183.4 \mathrm{ab}$ & $261.9 \mathrm{ab}$ & $191.6 \mathrm{ab}$ & $82.1 \mathrm{~cd}$ & $273.8 \mathrm{bc}$ \\
\hline A4-1 & - & - & - & - & $203.6 \mathrm{ab}$ & $263.3 \mathrm{ab}$ & $190.8 \mathrm{ab}$ & $98.2 \mathrm{~b}$ & $289.1 \mathrm{ab}$ \\
\hline A4-2 & $243.8 \mathrm{a}$ & $173.9 \mathrm{a}$ & $122.1 \mathrm{a}$ & $296.0 \mathrm{a}$ & $161.5 \mathrm{~b}$ & $238.7 \mathrm{~b}$ & $168.3 \mathrm{~b}$ & $81.8 \mathrm{~cd}$ & $250.2 \mathrm{c}$ \\
\hline
\end{tabular}

$\mathrm{A} 1:$ 小麦-大豆连作; A2: 小麦-甘薯连作; A3-1：小麦/玉米/大豆套作边行; A3-2: 小麦/玉米/大豆套作中行; A4-1: 小麦/玉米/甘 薯套作边行; A4-2: 小麦/玉米/甘薯套作中行。表中数据为 3 次重复的平均值。同一列中标以不同字母的值差异达 0.05 显著水平。 2006-2007 年度, 套作处理未按边行和中行分别取样, 因此只有一组数据。

A1: wheat-soybean cropping system; A2: wheat-sweet potato cropping system; A3-1: fringe rows of wheat/maize/soybean cropping system; A3-2: central rows of wheat/maize/soybean cropping system; A4-1: fringe rows of wheat/maize/sweet potato cropping system; A4-2: central rows of wheat/maize/sweet potato cropping system. Data are the averages of three replicates. Values followed by a different letter within each column are significantly different at the 0.05 probability level. In 2006-2007, wheat plants were sampled only from central rows of treatments $\mathrm{A} 3$ and $\mathrm{A} 4$. 
表 5 不同种植模式的小麦土壤总氮含量

Table 5 Total nitrogen content of wheat soil under various cropping systems (\%)

\begin{tabular}{|c|c|c|c|c|c|}
\hline \multirow{2}{*}{$\begin{array}{c}\text { 处理 } \\
\text { Treatment }\end{array}$} & \multicolumn{2}{|c|}{ 2006-2007 } & \multicolumn{3}{|c|}{ 2007-2008 } \\
\hline & $\begin{array}{c}\text { 开花期 } \\
\text { Flowering stage }\end{array}$ & $\begin{array}{c}\text { 成熟期 } \\
\text { Maturity stage }\end{array}$ & $\begin{array}{c}\text { 拔节期 } \\
\text { Jointing stage }\end{array}$ & $\begin{array}{c}\text { 开花期 } \\
\text { Flowering stage }\end{array}$ & $\begin{array}{c}\text { 成熟期 } \\
\text { Maturity stage }\end{array}$ \\
\hline A1 & $0.215 \mathrm{a}$ & $0.166 \mathrm{~b}$ & $0.286 \mathrm{a}$ & $0.252 \mathrm{a}$ & $0.234 \mathrm{ab}$ \\
\hline A2 & $0.210 \mathrm{a}$ & $0.160 \mathrm{~b}$ & $0.290 \mathrm{a}$ & $0.245 \mathrm{a}$ & $0.227 \mathrm{~b}$ \\
\hline A3-1 & - & - & $0.274 \mathrm{a}$ & $0.261 \mathrm{a}$ & $0.245 \mathrm{a}$ \\
\hline A3-2 & $0.202 \mathrm{a}$ & $0.190 \mathrm{a}$ & $0.271 \mathrm{a}$ & $0.257 \mathrm{a}$ & $0.242 \mathrm{a}$ \\
\hline A4-1 & - & - & $0.287 \mathrm{a}$ & $0.252 \mathrm{a}$ & $0.239 \mathrm{ab}$ \\
\hline A4-2 & $0.201 \mathrm{a}$ & $0.196 \mathrm{a}$ & $0.249 \mathrm{~b}$ & $0.249 \mathrm{a}$ & $0.229 \mathrm{~b}$ \\
\hline
\end{tabular}

A1：小麦-大豆连作; A2：小麦-甘薯连作; A3-1：小麦/玉米/大豆套作边行；A3-2：小麦/玉米/大豆套作中行; A4-1：小麦/玉米/甘 薯套作边行; A4-2: 小麦/玉米/甘薯套作中行。表中数据为 3 次重复的平均值。同一列中标以不同字母的值差异达 0.05 显著水平。 2006-2007 年度, 套作处理未按边行和中行分别取样, 因此只有一组数据。

A1: wheat-soybean cropping system; A2: wheat-sweet potato cropping system; A3-1: fringe rows of wheat/maize/soybean cropping system; A3-2: central rows of wheat/maize/soybean cropping system; A4-1: fringe rows of wheat/maize/sweet potato cropping system; A4-2: central rows of wheat/maize/sweet potato cropping system. Data are the averages of three replicates. Values followed by a different letter within each column are significantly different at the 0.05 probability level. In 2006-2007, wheat plants were sampled only from central rows of treatments $\mathrm{A} 3$ and $\mathrm{A} 4$.

表 6 不同种植模式对小麦土壤中 $\mathrm{NO}_{3}{ }^{-}-\mathrm{N}$ 和 $\mathrm{NH}_{4}{ }^{+}-\mathrm{N}$ 含量的影响

Table 6 Effect of cropping system on contents of $\mathrm{NO}_{3}{ }^{-}-\mathrm{N}$ and $\mathrm{NH}_{4}{ }^{+}-\mathrm{N}$ in wheat soil $\left(\mathrm{mg} \mathrm{kg}^{-1}\right)$

\begin{tabular}{|c|c|c|c|c|c|c|c|c|c|c|}
\hline \multirow{3}{*}{$\begin{array}{c}\text { 处理 } \\
\text { Treatment }\end{array}$} & \multicolumn{4}{|c|}{ 2006-2007 } & \multicolumn{6}{|c|}{ 2007-2008 } \\
\hline & \multicolumn{2}{|c|}{ 开花期 Flowering stage } & \multicolumn{2}{|c|}{ 成熟期 Maturity stage } & \multicolumn{2}{|c|}{ 拔节期 Jointing stage } & \multicolumn{2}{|c|}{ 开花期 Flowering stage } & \multicolumn{2}{|c|}{ 成熟期 Maturity stage } \\
\hline & $\mathrm{NO}_{3}^{-}-\mathrm{N}$ & $\mathrm{NH}_{4}{ }^{+}-\mathrm{N}$ & $\mathrm{NO}_{3}^{-}-\mathrm{N}$ & $\mathrm{NH}_{4}{ }^{+}-\mathrm{N}$ & $\mathrm{NO}_{3}^{-}-\mathrm{N}$ & $\mathrm{NH}_{4}{ }^{+}-\mathrm{N}$ & $\mathrm{NO}_{3}^{-}-\mathrm{N}$ & $\mathrm{NH}_{4}{ }^{+}-\mathrm{N}$ & $\mathrm{NO}_{3}^{-}-\mathrm{N}$ & $\mathrm{NH}_{4}{ }^{+}-\mathrm{N}$ \\
\hline A1 & $41.61 \mathrm{a}$ & $27.34 \mathrm{a}$ & $22.89 \mathrm{a}$ & $18.58 \mathrm{a}$ & $21.00 \mathrm{ab}$ & $27.35 \mathrm{abc}$ & $24.30 \mathrm{c}$ & $75.25 \mathrm{bc}$ & $14.50 \mathrm{~b}$ & $13.04 \mathrm{ab}$ \\
\hline A2 & $44.08 \mathrm{a}$ & $26.41 \mathrm{a}$ & $24.75 \mathrm{a}$ & $19.10 \mathrm{a}$ & $23.25 \mathrm{a}$ & $30.50 \mathrm{a}$ & $32.70 \mathrm{a}$ & $95.80 \mathrm{a}$ & $18.94 \mathrm{a}$ & $14.34 \mathrm{a}$ \\
\hline A3-1 & - & - & - & - & $15.95 \mathrm{~d}$ & $25.15 \mathrm{c}$ & $18.95 \mathrm{~d}$ & $61.15 \mathrm{c}$ & $9.80 \mathrm{e}$ & $11.46 \mathrm{~b}$ \\
\hline A3-2 & $37.32 \mathrm{~b}$ & $20.28 \mathrm{~b}$ & $12.48 \mathrm{~b}$ & $16.10 \mathrm{a}$ & $17.15 \mathrm{~cd}$ & $25.95 \mathrm{bc}$ & $20.70 \mathrm{~d}$ & $72.15 \mathrm{bc}$ & $11.18 \mathrm{de}$ & $12.10 \mathrm{~b}$ \\
\hline A4-1 & - & - & - & - & $19.85 \mathrm{bc}$ & $27.70 \mathrm{abc}$ & $24.50 \mathrm{c}$ & $80.40 \mathrm{ab}$ & $12.06 \mathrm{~cd}$ & $11.99 \mathrm{~b}$ \\
\hline A4-2 & $37.20 \mathrm{~b}$ & $19.04 \mathrm{~b}$ & $9.76 \mathrm{c}$ & $16.16 \mathrm{a}$ & $20.60 \mathrm{ab}$ & $29.15 \mathrm{ab}$ & $27.30 \mathrm{~b}$ & $92.35 \mathrm{a}$ & $14.01 \mathrm{bc}$ & $12.90 \mathrm{ab}$ \\
\hline
\end{tabular}

$\mathrm{A} 1$ : 小麦-大豆连作; $\mathrm{A} 2$ : 小麦-甘薯连作; $\mathrm{A} 3-1$ : 小麦/玉米/大豆套作边行; A3-2: 小麦/玉米/大豆套作中行; A4-1: 小麦/玉米/甘 薯套作边行; A4-2: 小麦/玉米/甘薯套作中行。表中数据为 3 次重复的平均值。同一列中标以不同字母的值差异达 0.05 显著水平。 2006-2007 年度, 套作处理未按边行和中行分别取样, 因此只有一组数据。

A1: wheat-soybean cropping system; A2: wheat-sweet potato cropping system; A3-1: fringe rows of wheat/maize/soybean cropping system; A3-2: central rows of wheat/maize/soybean cropping system; A4-1: fringe rows of wheat/maize/sweet potato cropping system; A4-2: central rows of wheat/maize/sweet potato cropping system. Data are the averages of three replicates. Values followed by a different letter within each column are significantly different at the 0.05 probability level. In 2006-2007, wheat plants were sampled only from central rows of treatments $\mathrm{A} 3$ and $\mathrm{A} 4$.

+ 花生间作系统中玉米根系分泌物可活化土壤难溶 性铁而提高土壤有效铁含量, 从而提高花生对铁的 吸收与利用 ${ }^{[25]}$ 。

本研究表明, 小麦/玉米/大豆和小麦/玉米/甘薯 套作模式相对于小麦-大豆及小麦-甘薯净作模式增 加了根系分泌有机酸总量和可溶性糖含量, 其中净 作模式中小麦以分泌乙酸为主, 而在套作模式中小 麦除分泌乙酸外, 还有较强的分泌柠檬酸和琥珀酸 能力。这与郝艳茹等 ${ }^{[24]}$ 和孙否等 ${ }^{[6]}$ 的研究结果基本 一致。除套作影响根系分泌有机酸外, 前作茬口对
后作小麦根系分泌能力也有较大影响, 大豆茬口的 小麦根系分泌有机酸总量和可溶性糖含量明显高于 甘薯茬口。出现上述现象的原因可能与大豆固氮作 用增强了对土壤的培肥作用和小麦与玉米共生体系 中的种间氮素促进作用有关。两年换茬轮作试验表 明，套作和大豆茬口处理中，小麦根系干重和根系 活力显著提高, 小麦生长得到改善, 从而促进了根 系分泌物的产生。另外, 小麦边行的根系分泌有机 酸与可溶性糖的含量高于中行, 说明与玉米套作促 进了小麦的根系分泌能力或玉米通过根系交互作用 
把自身的根系分泌物运移到小麦根区，以改善小麦 根际生态环境, 促进其生长和养分的吸收。其作用 机制有待进一步验证。

3.2 套作条件下根系分泌物与植物生长环境和 养分吸收的关系

根系分泌物通过改变植物根际 $\mathrm{pH}$ 值、氧化还原 状态, 或者通过鳌合和还原作用将土壤中难溶的矿 质转化为可被植物吸收利用的状态, 间接或直接地 影响土壤养分的有效性 ${ }^{[26-28]}$ 。本研究表明, 小麦茎 叶和籽粒的吸氮量表现为套作高于净作, 大豆茬口 高于甘薯茬口, 这与小麦自身根系分泌能力有关, 另外套作对土壤有效氮有调节作用。小麦与玉米共 生前, 套作和大豆茬口处理的土壤总氮含量相对比 净作和甘薯茬口高, 促进了小麦植株从土壤中吸取 较多养分, 表现为土壤总氮含量降低, 植株吸氮量 增加和根系生长能力加强; 随着植株生长能力的增 强, 根系分泌作用也随之增强, 继而改善环境, 例 如小麦/玉米/大豆套作模式降低了土壤湿度和 $\mathrm{pH}$ 值, 从而确保冬季小麦在外界环境温度较低时, 土壤保
持相对较高温度, 利于土壤酶活性和微生物活动增 强, 调节土壤中氮素的转化过程, 使其向有利于作 物吸收氮形态的方向进行。本研究中, 在开花期(小 麦与玉米的共生期), 小麦植株的氮素含量和土壤总 氮含量均以套作和大豆茬口较高, 而 $\mathrm{NO}_{3}{ }^{-}-\mathrm{N}$ 和 $\mathrm{NH}_{4}{ }^{+}-\mathrm{N}$ 含量则相应降低。相关分析(表 7)表明, 开花 期小麦根系分泌有机酸总量和可溶性糖含量分别与 小麦根系干重、植株吸氮量、土壤总氮含量呈显著 正相关, 与 $\mathrm{NO}_{3}{ }^{-}-\mathrm{N}$ 和 $\mathrm{NH}_{4}{ }^{+}-\mathrm{N}$ 含量呈显著负相关, 而植株吸氮量又与根系活力、根系干重及土壤总氮 含量呈显著正相关, 与 $\mathrm{NO}_{3}{ }^{-}-\mathrm{N}$ 和 $\mathrm{NH}_{4}{ }^{+}-\mathrm{N}$ 含量呈显 著负相关。在小麦/玉米/大豆套作体系中, 如何通过 种间互惠互利作用来发挥根系分泌物对作物生长环 境的改善和土壤养分的活化, 以增强作物对环境的 生态适应性, 促进根系对养分和水分的吸收, 是实 现套作增产、提高肥料利用效率的有力保障。在套 作体系中, 尤其是小麦/玉米/大豆套作模式在微区 轮作效应下，根系分泌物如何影响土壤微生物和土 壤酶活性还有待进一步研究。

表 7 小麦根系分泌物与根系活力、土壤 $\mathbf{N}$ 含量及植株吸氮量的相关系数

Table 7 Correlation coefficients between root exudates and root activity, soil nitrogen content, and nitrogen uptake

\begin{tabular}{|c|c|c|c|c|c|c|c|}
\hline & $\begin{array}{c}\text { 有机酸总量 } \\
\text { Organic acid } \\
\text { content }\end{array}$ & $\begin{array}{c}\text { 可溶性糖含量 } \\
\text { Soluble sugar } \\
\text { content }\end{array}$ & $\begin{array}{c}\text { 根系活力 } \\
\text { Root activity }\end{array}$ & $\begin{array}{c}\text { 根系干重 } \\
\text { Dry matter } \\
\text { weight of roots }\end{array}$ & $\begin{array}{c}\mathrm{NO}_{3}^{-}-\mathrm{N} \text { 含量 } \\
\mathrm{NO}_{3}^{-}-\mathrm{N} \text { content }\end{array}$ & $\begin{array}{c}\mathrm{NH}_{4}^{+}-\mathrm{N} \text { 含量 } \\
\mathrm{NH}_{4}^{+}-\mathrm{N} \text { content }\end{array}$ & $\begin{array}{c}\text { 土壤总氮含量 } \\
\text { Total N content } \\
\text { in soil }\end{array}$ \\
\hline $\begin{array}{l}\text { 可溶性糖含量 } \\
\text { Soluble sugar content }\end{array}$ & $0.950^{* *}$ & & & & & & \\
\hline $\begin{array}{l}\text { 根系活力 } \\
\text { Root activity }\end{array}$ & 0.608 & 0.570 & & & & & \\
\hline $\begin{array}{l}\text { 根系干重 } \\
\text { Dry matter weight of roots }\end{array}$ & $0.717^{*}$ & $0.838^{*}$ & 0.682 & & & & \\
\hline $\begin{array}{l}\mathrm{NO}_{3}^{-}-\mathrm{N} \text { 含量 } \\
\mathrm{NO}_{3}^{-}-\mathrm{N} \text { content }\end{array}$ & $-0.733^{*}$ & $-0.822^{*}$ & -0.651 & $-0.944^{* *}$ & & & \\
\hline $\begin{array}{l}\mathrm{NH}_{4}^{+}-\mathrm{N} \text { 含量 } \\
\mathrm{NH}_{4}^{+}-\mathrm{N} \text { content }\end{array}$ & $-0.770^{*}$ & $-0.810^{*}$ & $-0.834^{*}$ & $-0.952^{* *}$ & $0.945^{* *}$ & & \\
\hline $\begin{array}{l}\text { 土壤总氮含量 } \\
\text { Total } N \text { content in soil }\end{array}$ & $0.815^{*}$ & $0.836^{*}$ & 0.718 & $0.907^{*}$ & $-0.975^{* *}$ & $-0.963^{* *}$ & \\
\hline $\begin{array}{l}\text { 植株吸氮量 } \\
\text { N uptake }\end{array}$ & $0.899^{* *}$ & $0.925^{* *}$ & $0.781^{*}$ & $0.935^{* *}$ & $-0.900^{* *}$ & $-0.962^{* *}$ & $0.934^{* *}$ \\
\hline
\end{tabular}

$$
{ }^{*} P<0.05 ;{ }^{* *} P<0.01 \text {. }
$$

\section{4 结论}

小麦/玉米/大豆套作体系较小麦/玉米/甘薯、小 麦-大豆、小麦-甘薯种植方式更有利于小麦根系分泌 有机酸及可溶性糖, 分泌的有机酸在拔节期以柠檬 酸为主, 占有机酸总量的 $31.7 \% \sim 55.1 \%$, 开花期以 乙酸为主，占有机酸总量的 $33.3 \% \sim 78.3 \%$; 小麦/玉 米/大豆套作体系通过根系的分泌作用降低了小麦 根际土壤湿度、 $\mathrm{pH}$ 和 $\mathrm{NO}_{3}{ }^{-}-\mathrm{N} 、 \mathrm{NH}_{4}{ }^{+}-\mathrm{N}$ 含量, 使小
麦根系生长环境改善, 提高根系活力和根系干重, 最终促进小麦植株对氮素的吸收。

\section{References}

[1] Lynch J M, Whipps J M. Substrate flow in the rhizosphere. Plant Soil, 1990, 129: 1-10

[2] Tu S-X(涂书新), Guo Z-F(郭智芬). The root exudation of grain amaranth and its role in release of mineral potassium. Acta Agric Nucl Sin (核农学报), 1999, 13(5): 305-311 (in Chinese with 
English abstract)

[3] Li T-X(李廷轩), Ma G-R(马国瑞), Zhang X-Z(张锡洲), Wang $\mathrm{C}-\mathrm{Q}$ (王昌全). Change characteristics of organic acid and amino acid in root exudates in different grain amaranth genotypes. Plant Nutr Fert Sci (植物营养与肥料学报), 2005, 11(5): 647-653 (in Chinese with English abstract)

[4] Zhang F-S(张福锁). New Trends of Soil and Plant Nutrition (土 壤与植物营养研究新动态). Beijing: Beijing Agricultural University Press, 1992. pp 64-70 (in Chinese)

[5] Rice E J, Pancholy S K. Inhibition of nitrification by climax ecosystems: Inhibitors other than tannins. Am J Bot, 1974, 61: 1095-1103

[6] Sun L(孙否), Chen B-L(陈兵林), Zhou Z-G(周治国). Effects of root exudates on available soil nutrition, micro-organism quantity of $B t$ cotton in wheat-cotton intercropping system. Cotton Sci (棉 花学报), 2007, 19(1): 18-22 (in Chinese with English abstract)

[7] Thackray D J, Wratien S D, Edwards P J, Niemeyer H M. Resistance to the aphids sitobion avenae and rhopal osiphum padi in Graminea in relation to hydroxamic levels. Ann Appl Biol, 1990, 116: $573-582$

[8] Lipton D S, Blanchar R W, Blevins D G. Citrate, malate, and succinate concentration in exudates from P-sufficient and P-stressed Medicago sativa L. seedings. Plant Physiol, 1987, 85: 315-317

[9] Tu S-X(涂书新), Sun J-H(孙锦荷), Guo Z-F(郭智芬), Gu F(谷 峰). On relationship between root exudates and plant nutrition in rhizosphere. Soil Environ Sci (土壤与环境), 2000, 9(1): 64-67 (in Chinese with English abstract)

[10] Chen L-C(陈龙池), Liao L-P(廖利平), Wang S-L(汪思龙), Xiao F-M(肖复明). A review for research of root exudates ecology. Chin J Ecol (生态学杂志), 2002, 21(6): 57-62(in Chinese with English abstract)

[11] Zhang F-S(张福锁). Enviroment Stress and Plant Rhizoshpere Nutrition (环境胁迫与植物根际营养). Beijing: China Agriculture Press, 1998. pp 16-56 (in Chinese)

[12] Yong T-W(雍太文), Ren W-J(任万军), Yang W-Y(杨文铎), Fan G-Q(㚞高琼). Meaning and characteristic and cultivation technique of "wheat/maize/soybean" relay cropping system. Gengzuo yu Zaipei (耕作与栽培), 2006, (6): 78-81 (in Chinese)

[13] Yong T-W(雍太文), Yang W-Y(杨文钰), Ren W-J(任万军), Fan $\mathrm{G}-\mathrm{Q}$ (樊高琼), Xiang D-B(向达兵). Analysis of the nitrogen transfer, nitrogen uptake and utilization in the two relay-planting systems. Sci Agric Sin (中国农业科学), 2009, 42(9): 3170-3178 (in Chinese with English abstract)

[14] Yong T-W(雍太文), Yang W-Y(杨文钰), Fan G-Q(樊高琼), Zhang Y-F(张亚飞). Study on the annual balance application of nitrogen fertilizer in the relay-cropping system of "wheat/maize/ soybean”. Soil Fert China (中国土壤与肥料), 2009, (3): 31-35 (in Chinese with English abstract)

[15] Yong T-W(雍太文), Yang W-Y(杨文钰), Wang X-C(王小春), Fan G-Q(樊高琼). Study on the nitrogen uptake and utilization and interspecies reciprocity in the two relay-planting systems. $J$
Sichuan Agric Univ (四川农业大学学报), 2009, 27(2): 167-172

(in Chinese with English abstract)

[16] Bao S-D(鲍士旦). Analysis Methods for Soil Agrochemistry (土 壤农化分析). Beijing: Agriculture Press, 1987. pp 191-203 (in Chinese)

[17] Hu X-Y(胡学玉), Li X-Y(李学垣), Xie Z-C(谢振翅). Differences of $\mathrm{Zn}$ uptake in various pakchoi cultivars relationship between Zn uptake root exudates. Plant Nutr Fert Sci (植物营养与 肥料学报), 2002, 8(2): 234-238(in Chinese with English abstract)

[18] Hao J-J(郝建军), Kang Z-L(康宗利), Yu Y(于洋). Experimental Techniques for Plant Physiology (植物生理学实验技术). Beijing: Chemical Industry Press, 2007. pp 89-112 (in Chinese)

[19] Liu H-S(刘洪升), Song Q-H(宋秋华), Li F-M(李凤民). The role of root exudates on rhizosphere mineral nutrition and rhizosphere micro-organisms. Acta Bot Boreali-Occident Sin (西北植物学报), 2002, 22(3): 693-702 (in Chinese with English abstract)

[20] Li Z-H(李志洪), Chen D(陈丹), Sun X-Q(孙晓秋), Dou S(窦森). Effects of phosphorus deficiency on excretion of organic acids for different maize genotypes and mobilization of undissolved phosphorus. Plant Physiol Commun (植物生理学通讯), 1999, 35(6): 455-457 (in Chinese with English abstract)

[21] Zhang X-Z(张锡洲), Li T-X(李廷轩), Wang Y-D(王永东). Relationship between growth environment and root exudates of plants. Chin J Soil Sci (土壤通报), 2007, 38(4): 785-789 (in Chinese with English abstract)

[22] Roemheld V, Marschner H. Genotypes differences among graminaceous in release of phytosiderophores and uptake of iron phytosiderophores. Plant Soil, 1990, 123: 147-153

[23] Strom L, Olsson T, Tyler G. Difference between calcifuge and acidfuge plants in root exudation of low-molecular organic acids. Plant Soil, 1994, 167: 239-245

[24] Hao Y-R(郝艳茹), Lao X-R(劳秀荣), Sun W-H(孙伟红), Peng $\mathrm{S}-\mathrm{L}$ (彭少麟). Interaction of roots and rhizophere in the wheat-maize intercropping system. Rural Eco-Environ (农村生 态环境), 2003, 19(4): 18-22 (in Chinese with English abstract)

[25] Zuo Y-M(左元梅), Chen Q(陈清), Zhang F-S(张福锁). The mechanisms of root exudates of maize in improvement of iron nutrition of peanut in peanut/maize intercropping system by ${ }^{14} \mathrm{C}$ tracer technique. Acta Agric Nucl Sin (核农学报), 2004, 18(1): 43-46 (in Chinese with English abstract)

[26] Nardi S, Concheri G, Pizzeghello D, Sturaro A, Rella R, Parvoli G. Soil organic matter mobilization by root exudates. Chemosphere, 2000, 41: 653-658

[27] Marschener H. Role of root growth, arbuscular mycorrhiza, and root exudates for the efficiency in nutrient acquisition. Field Crops Res, 1998, 56: 203-207

[28] Sun L(孙否), Chen B-L(陈兵林), Zhou Z-G(周治国). Effect of allelopathic substance from wheat root zones on the growth of cotton seedling in wheat-cotton inter-planting system. Cotton Sci (棉花学报), 2006, 18(4): 213-217 (in Chinese with English abstract) 